\title{
La linguistique populaire et les pseudo-savants
}

\section{Patrick Sériot, Elena Bulgakova et Andreja Eržen}

\section{(2) OpenEdition}

\section{Journals}

Édition électronique

URL : http://journals.openedition.org/pratiques/1220

DOI : 10.4000/pratiques. 1220

ISSN : 2425-2042

\section{Éditeur}

Centre de recherche sur les médiations (CREM)

\section{Édition imprimée}

Date de publication : 15 décembre 2008

Pagination : 149-162

\section{Référence électronique}

Patrick Sériot, Elena Bulgakova et Andreja Eržen, « La linguistique populaire et les pseudo-savants », Pratiques [En ligne], 139-140 | 2008, mis en ligne le 15 décembre 2008, consulté le 08 mars 2021. URL : http://journals.openedition.org/pratiques/1220; DOI : https://doi.org/10.4000/pratiques.1220

(C) Tous droits réservés 


\title{
La linguistique populaire et les pseudo-savants
}

\section{Patrick Sériot, Elena Bulgakova, Andreja Eržen}

\author{
Université de Lausanne
}

Dans L'Etoile rouge (1908), le théoricien du futur Proletkult A. Bogdanov (1873-1928) imagine une humanité martienne ayant accompli la révolution socialiste sur la planète Mars, enfin délivrée de ses divisions, et ne parlant désormais qu'une seule langue, harmonieuse et parfaite, en complète adéquation avec le réel à dire, ou plutôt à nommer. A la fin du $\mathrm{XIX}^{\mathrm{e}}$ et au début du $\mathrm{XX}^{\mathrm{e}}$ siècle, des centaines de projets de langue universelle partageaient le rêve de L. Zamenhof, l'inventeur de l'espéranto : venger Babel, réunir ce que Dieu avait dissocié, combler la béance du signe, qui nous disjoint du Tout et nous sépare des autres ${ }^{(1)}$.

Au début du XXI $\mathrm{XI}^{\mathrm{e}}$ siècle, supposé être celui de la «mondialisation », les choses ont étonnamment changé. Sans doute parce que l'anglais en joue déjà le rôle, personne n'invente plus de langue universelle. Une autre anthropologie implicite s'est mise en place, une autre vision de l'humanité s'est déployée : les humains ne sont pas seulement des êtres de langage, ce sont avant tout des êtres de langue. L'écologie linguistique dit « halte à la mort des langues!», faisant référence aux espèces naturelles en voie de disparition. Or, il est un endroit du monde où, loin de disparaître, les langues au contraire poussent comme champignons après la pluie. En Europe centrale, orientale et balkanique, en l'espace de quelques années, des objets nouveaux sont apparus sur le marché des langues. En juin 2006 le parlement du Monténégro ayant tout juste proclamé son indépendance, adopte une déclaration affirmant que « la langue du Monténégro est le monténégrin » [crnogorski jezik]. Mais l'existence de cette langue, pourtant officielle, en tant que différente du serbe n'est pas reconnue par tous les linguistes du Monténégro. A l'inverse, l'existence du ruthène [rusyns ' $k a$ mova] en tant que différent de l'ukrainien est reconnue par le gouvernement slovaque mais pas par le gouvernement ukrainien ( $c f$. Sériot, 2006).

Le discours sur la langue comme fondement d'une interrogation sur l'identité collective et la justification politique de l'existence d'un Etat indépendant tend à enfler démesurément à l'Est de l'Europe. C'est à une variété peu connue de ce discours sur la langue que va s'attacher cet article, fruit du travail d'une équipe de l'Université de Lausanne : la linguistique populaire de pseudo-savants.

Le corpus est immense, à la mesure de l'instabilité identitaire des nouveaux

(1) Sur l'image de la société humaine implicite dans les projets de langues universelles de cette époque, voir Michéa, 1979. 
Etats de l'Est de l'Europe, issus d'empires récents ou anciens, laminés par des guerres et des redécoupages incessants de frontières. Nous sélectionnons dans ce corpus le thème de l'invention des ancêtres glorieux, révélés par le jeu d'une étymologie fantastique. Il est impossible de dire, à ce stade de l'enquête, que ces textes soient représentatifs, ils ont simplement pour eux le fait d'exister, d'être lus, commentés, vendus en librairies, parfois commentés à la télévision.

Les auteurs sont, selon les pays, simples marginaux illuminés ou universitaires connaissant des succès de librairie. Que des rêveurs monomaniaques obsédés par l'idée du complot du silence, qu'ils soient savants amateurs, fervents patriotes, fous du langage, enseignants au service d'un projet politique, inventent ou propagent des mythes identitaires linguistiques en les présentant comme des découvertes scientifiques n'a en soi rien de nouveau. On se lasserait même vite de lire leurs élucubrations qui ne regardent qu'eux-mêmes si ce n'était l'étonnante similitude entre les thèmes abordés par des chercheurs isolés, en pure ignorance réciproque, vivant parfois à des milliers de kilomètres les uns des autres. Comment se fait-il que des gens qui pensent pis que pendre de leurs voisins disent en réalité la même chose qu'eux, la même souffrance, le même désir d'être reconnus, respectés, estimés? C'est à reconstituer ce texte prototypique qu'est consacré le présent travail.

Il reste à inventer une dénomination pour cet étonnant discours sur la langue : linguistique marginale, nationaliste, ethniciste ? Linguistique révisionniste ferait bien l'affaire, si ce mot n'était pas aussi chargé de connotations. On choisira « linguistique du nouveau paradigme », du nom d'un courant répandu en Finlande et en Estonie, ayant pour but de dénier toute existence à la grammaire historico-comparative (qui affirme une origine commune au hongrois et au finnois) et d'affirmer que les liens acquis entre le suédois et le finnois sont plus forts et plus authentiques (cf. Smit, 2001).

Il reste à savoir pourquoi, à un certain moment, des gens se mettent à croire à des fables savantes. Ces textes délirants ne sont pas en général le résultat d'une propagande d'Etat, personne n'oblige ces pseudo-savants à écrire ce qu'ils écrivent, aucune pression ne s'exerce, aucune promesse de carrière académique n'est à la clé, ils n'en retirent aucun avantage personnel. Ils y croient eux-mêmes, voilà l'expression d'une profonde souffrance identitaire.

Nous prenons pour corpus des textes de linguistique qu'on rangera, à la manière des éditeurs du recueil La linguistique fantastique (Auroux et al., 1983) dans un domaine marginal et appartenant à une linguistique à la fois populaire et fantastique, les deux termes étant ici inséparables. Mais ici la situation est particulière : la linguistique fantastique n'est pas nécessairement rejetée par l'institution, et la linguistique populaire est parfois au centre du discours savant.

La linguistique populaire et fantastique en Europe centrale et orientale forme un ensemble de variantes à partir d'une matrice commune, variantes dont les revendications identiques sont mutuellement exclusives. Ainsi, on lit que les ancêtres des Slovènes parlaient la langue vénète, totalement différente de celle des Slaves, la même que celle des Etrusques et des Basques, mais que ceux des Serbes parlaient la langue illyrienne, qui est la même que celle des Etrusques et des Basques. Les ancêtres des Albanais parlaient la langue illyrienne, la même que celle des Etrusques et des Basques (et les Grecs ont détruit toute trace de l'antique civilisation illyrienne, c'est-à-dire albanaise). Les ancêtres des Ukrainiens parlaient une langue qui a donné naissance au sanskrit, mais aussi à l'étrusque. Les Ukrainiens sont les seuls vrais Slaves (les Russes ne sont alors qu'un conglomérat hybride de Tatars et de Finno-Ougriens), mais les Serbes sont dans les Balkans de toute éternité avant les Albanais, qui sont dans les Balkans de toute éternité avant les Grecs. 
Le discours de l'exclusion a ainsi ceci de hautement singulier de reposer sur une totale similitude d'arguments. Anne-Marie Thiesse, dans son remarquable ouvrage La création des identités nationales. Europe 18e-20e s. (2001), montre comment un travail de comparaison des productions identitaires au XIX ${ }^{\mathrm{e}}$ siècle (arts et traditions populaires, costume folklorique, littérature populaire, etc.) fait apparaître que

«[...] le résultat de la fabrication collective des identités nationales n'est pas un moule unique, mais bien plutôt, selon l'expression provocatrice du sociologue Orvar Löfgren ${ }^{(2)}$, une sorte de kit en "do-it-yourself” : une série de déclinaisons de l'“âme nationale" et un ensemble de procédures nécessaires à leur élaboration. On sait bien aujourd'hui établir la liste des éléments symboliques et matériels que doit présenter une nation digne de ce nom : une histoire établissant la continuité avec les grands ancêtres, une série de héros parangons des vertus nationales, une langue, des monuments culturels, un folklore, des hauts lieux et un paysage typique, une mentalité particulière, des représentations officielles - hymne et drapeau - et des identifications pittoresques - costume, spécialités culinaires ou animal emblématique. » (Thiesse, 2001, pp. 13-14)

Il reste à expliciter les différences entre le discours identitaire en Europe occidentale au début du XIX ${ }^{\mathrm{e}}$ siècle (trouver des ancêtres communs aux Auvergnats et aux Normands, aux Siciliens et aux Piémontais) et celui qui domine en Europe orientale et balkanique deux siècles plus tard (trouver des ancêtres différents aux Serbes et aux Croates, aux Macédoniens et aux Bulgares, aux Ruthènes et aux Ukrainiens, aux Moldaves et aux Roumains).

\section{La théorie du complot : une linguistique du ressentiment}

Dès avant l'indépendance des nouveaux Etats d'Europe centrale et orientale apparaissent de nouvelles théories sur les origine de la nation et de sa langue, focalisées sur une réinterprétation de l'histoire primitive, «primordiale» de la communauté. Cet intérêt est tout à la fois un refus de l'histoire évolutionniste marxiste et la conséquence de la frustration d'intellectuels de ces « jeunes nations » envers les nations que Hegel qualifia d'« historiques ». Dans ce discours nationaliste, l'ancien est valorisé aux dépens du nouveau. Une nation se doit d'avoir une langue particulière dont l'origine remonte à la nuit des temps, comme elle doit avoir une longue tradition, des monuments, des contes, des chants nationaux que le monde entier doit bientôt découvrir. La fascination devant les mythes les plus antiques, les reliques nationales, s'exprime dans la multiplication des musées historiques, qui présentent une interprétation ethno-centrée de l'histoire de l'Europe centrale et orientale, où le passé national est instrumentalisé pour prouver que la nation à laquelle on appartient est au même niveau, sur le même plan de dignité que les autres, les « grandes ». Le discours sur la langue est une partie fondamentale de cette entreprise de légitimation des nouveaux Etats.

Les promoteurs de ce qu'on peut appeler une linguistique du ressentiment se sentent rejetés par la « science officielle », ce qui renforce en eux la théorie du complot du silence et le sentiment que, si leurs idées sont repoussées, c'est la preuve qu'elles sont vraies ${ }^{(3)}$. La linguistique officielle est considérée comme inefficace, elle est accusée de ne pas avoir changé de position, même après l'indépendance. A cet égard, une comparaison s'avère utile entre l'Ukraine et la Slovénie, deux pays si-

(2) Löfgren, 1989.

(3) Sur la notion de ressentiment, cf. Angenot, 1996. 
tués à $1500 \mathrm{~km}$ l'un de l'autre, où des auteurs, en toute ignorance réciproque, utilisent des arguments étonnament semblables :

\section{Ukraine}

- Les chercheurs contemporains hésitent à remettre en question certaines thèses qui concernent l'histoire ancienne (de l'Ukraine). (Gubernačuk, 2002, p. 4) - Même au temps de l'indépendance de l'Ukraine notre science officielle n'a pas changé son attitude envers les monuments écrits proto-ukrainiens, elle affirme qu'il n'existe aucune source prouvant l'origine ancienne de la langue ukrainienne. (ibid., p. 65)

- La position de la linguistique officielle ukrainienne envers l'histoire de la langue ukrainienne est la conséquence de la domination des régimes de la Russie (rossijskix) sur les terres ukrainiennes. (ibid., p. 77)

\section{Slovénie}

- La théorie selon laquelle les Slovènes sont venus au $\mathrm{VI}^{\mathrm{e}}$ siècle est une invention. Les historiens parlent seulement des Slaves. Même les archéologues admettent qu'il n'y a pas de preuves archéologiques. (Tomažič, 2003, p. 8).

- Les inscriptions des Vénètes sont des documents uniques, qui représentent la langue slovène ancienne. Aucune nation européenne, sauf les Grec, n'en a de si ancienne. (ibid., p. 9)

- La colonisation du territoire de la Slovénie par les Vénètes entre 1200 et 1000 avant J.-C. marque le commencement de l'ethnie slovène. Le plus probable c'est que les Vénètes sont venus en Italie depuis la Slovénie. (ibid., p. 39)

- Les chercheurs reconnus ne sont que des «personnes dressées par le régime». (Verbovšek, 2004, p. 171)

\section{Une linguistique du refus}

2.1. La linguistique du nouveau paradigme est un refus du monogénisme comme du polygénisme, du diffusionnisme comme de l'évolutionnisme.

Les caractéristiques communes de ces textes sont les suivantes :

- critique de la science « officielle», qui est mauvaise, avec, en contrepartie, la promotion d'une science « entièrement nouvelle »;

- recherche des origines de la communauté nationale dans les temps les plus reculés ;

- négation d'une communauté d'origine avec les autres nations (par exemple, négation de l'origine slave des Slovènes, ou déni par les Ukrainiens de l'origine slave des Russes) ;

- évolution linéaire de la langue (ni divergence, ni convergence) ou caractère stable de la langue.

On peut donc y voir un élément radicalement fixiste, anti-historiciste ${ }^{(4)}$, proche du créationnisme des théories anti-darwiniennes en biologie.

La linguistique du nouveau paradigme est une science du refus, qui s'inscrit dans la lignée générale du discours de réfutation.

Ainsi, dans le cas de l'Ukraine, l'historiographie de l'époque stalinienne a stabilisé une théorie datant de la fin du XIX ${ }^{\mathrm{e}}$ siècle, à savoir l'idée d'une communauté culturelle d'un peuple « vieux-russe » (drevnerusskaja narodnost') parlant une langue vernaculaire commune : le « vieux-russe» (drevnerusskij jazyk). Cette conception d'un peuple « vieux-russe » à l'origine des trois peuples-frères que sont les « Grands-

(4) Cet aspect anti-historiciste mériterait une étude approfondie, car on a pu qualifier les thèses nationalistes justement d'historicistes au sens que donne K. Popper à ce mot. 
Russes », les Biélorusses et les Ukrainiens (appelés «Petits-Russiens » par certains auteurs de Russie méridionale dès le milieu du XIX ${ }^{\mathrm{e}}$ siècle) était encore postulée par l'historien soviétique V. Mavrodin en 1946. Dans son livre La Russie ancienne (origine du peuple russe et formation de l'Etat de Kiev), il affirmait que

La Russie kiévienne ${ }^{(5)}$ est l'étape initiale de trois peuples-frères slaves, qui ont pour unique ancêtre le peuple russe de l'époque de la Russie kiévienne, le peuple russe ancien. (Mavrodin, 1946, p. 310)

La langue de la Russie kiévienne était le « russe commun» (Filin, 1972, p. 61). La langue ukrainienne s'était donc formée, sur la base des parlers vieux-russes du sud, vers le XII ${ }^{\mathrm{e}}$-XIII ${ }^{\mathrm{e}}$ siècles (Filin, ibid.). Il s'agissait ainsi d'une langue de périphérie par rapport au centre constitué par le russe.

L'académie des sciences de l'Ukraine actuelle défend une théorie différente, à savoir qu'à l'époque de la Russie kiévienne il y avait déjà trois unités ethniques différentes, qui ont donné ensuite naissance à trois nations : russe, ukrainienne et biélorusse. Le schéma ci-dessous illustre les origines de la langue ukrainienne selon les deux théories :

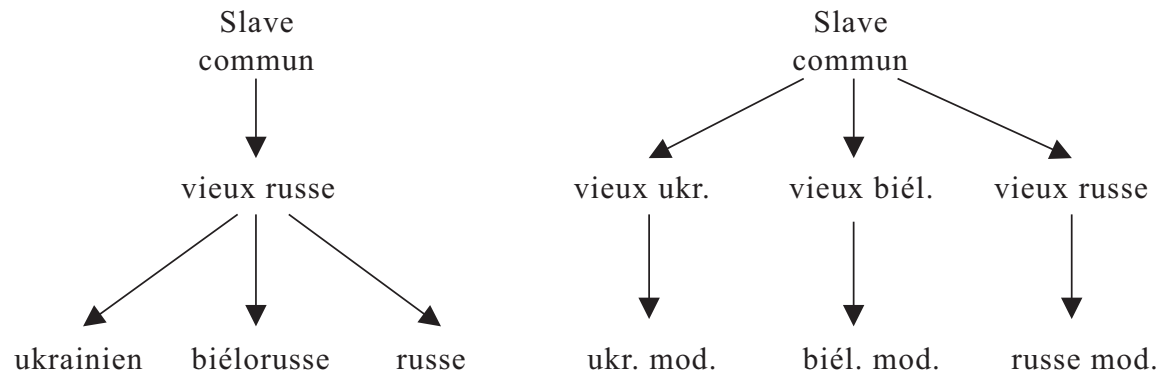

On va voir que les linguistes ukrainiens du nouveau paradigme rejettent le premier point de vue, mais ne partagent pas non plus entièrement le second, dont ils proposent une version radicalisée.

En Slovénie, il s'agit du rejet de la théorie « officielle » de la formation de la nation slovène, selon laquelle les Slaves seraient arrivés dans les Alpes orientales au $\mathrm{VI}^{\mathrm{e}}$ siècle. La « Nouvelle théorie » s'attache à la forme du nom par lequel les Autrichiens appelaient les habitants slavophones de Carinthie, Carniole et Styrie : die Wenden. Rattachant ce nom aux Veneti des auteurs latins, elle explique que ces Vénètes sont un peuple qui occupait le territoire de l'actuelle Slovénie depuis des temps immémoriaux, c'est-à-dire bien avant que les Celtes ne fassent leur apparition en Europe, et dont on trouve des vestiges archéologiques dans la culture des cimetières d'urnes. Cette théorie autochtoniste rejette l'appartenance de la langue slovène à la famille slave, essentiellement pour affirmer la différence entre les Slovènes et les Serbes (les Croates ne représentant pas une aussi grande menace). «Slave» est ainsi synonyme de barbare ${ }^{(6)}$.

(5) On appelle Russie kiévienne l'état féodal slave oriental constitué d'un réseau de villes gouvernées par des princes unis par des liens de parenté, depuis l'arrivée des chefs scandinaves au IX ${ }^{\mathrm{e}}$ siècle jusqu'au début du joug tatar en 1240. Cet Etat contrôlait les voies fluviales navigables entre la Baltique et la Mer Noire.

(6) Les théories autochtonistes correspondent à ce qu'on appelait dans la Roumanie de Ceauşescu et dans la Roumanie actuelle encore le protochronisme: les Roumains étaient là avant les Hongrois, avant les Slaves et avant les Romains. 
A première vue, le nouveau paradigme en Ukraine prend des formes différentes, puisque les Ukrainiens y sont parfois présentés comme les seuls vrais Slaves. Pourtant, le tableau revient vite au même, puisque, pour un philologue comme S. Gubernačuk, il n'y a jamais eu de communauté slave, mais des peuples slaves concrets. Dans son livre, Gubernačuk va plus loin que les linguistes et historiens contemporains qui nient l'existence de la communauté vieux-russe et de la langue vieux-russe, il nie l'existence de la communauté slave en général. Pour lui, l'idée même que certaines langues dérivent d'autres est fausse. Un de ses principaux arguments est qu'il n'y a pas de langues qui apparaissent maintenant, mais seulement des langues qui meurent. Les indéniables ressemblances entre les langues slaves actuelles sont expliquées par le voisinage, par une longue période de contacts linguistiques. Cet hyper-diffusionnisme ressemble beaucoup à la théorie de N. Troubetzkoy dans son article posthume de 1939: «Gedanken über das Indogermanenproblem », dont la thèse essentielle est que les langues indo-européennes ne forment pas une famille au sens génétique, mais se sont rapprochées par convergence. Les traits indo-européens communs sont donc acquis, et non pas hérités. Comme Troubetzkoy et N. Marr, Gubernačuk pense que la théorie indo-européenne est le fruit d'une pensée impérialiste venue d'Angleterre et d'Allemagne, mais Gubernačuk y ajoute la Russie (Gubernačuk, 2002, p. 11).

Les linguistes S. Gubernačuk et $\mathrm{O}$. Riznyčenko soutiennent une hypothèse strictement polygéniste pour l'ukrainien, mais appliquent au russe et au biélorusse la théorie de la corruption, ou dégradation à partir d'un rameau initial ${ }^{(7)}$. En effet, pour eux, la langue de la Russie de Kiev était le "vieil-ukrainien » et non le «vieux-russe » (Riznyčenko, 2001, p. 31). Avant l'indépendance de l'Ukraine, les « linguistes impérialistes » [= les linguistes russes] ont répandu une pseudo-théorie selon laquelle il aurait existé une langue « vieux-russe » à partir de laquelle se seraient formées ultérieurement les trois langues sœurs. Or, pour Riznyčenko, il n'y a jamais eu d'ancêtre commun au russe, au biélorusse et à l'ukrainien. Quant au slavon, c'était une langue artificielle, ayant servi, en quelque sorte, de base pour la formation du russe actuel. Riznyčenko ajoute un élément nouveau à la théorie polygéniste de Gubernačuk : le russe (qu'il appelle le moscovite) et le biélorusse résultent de la corruption du vieil-ukrainien par le finno-ougrien dans le cas du russe et par le balte dans le cas du biélorusse (ibid., p. 31).

On voit ainsi une hésitation, ou un amalgame entre trois modèles, ou plus exactement trois cadres de pensée : polygénèse radicale (les langues actuelles ont toutes existé " depuis le début»), diffusionisme (les ressemblances sont acquises par contact) et dégradation (corruption à partir d'un état de pureté initiale). Ces trois modèles peuvent coexister chez un même auteur à quelques pages ou lignes d'intervalle. Mais l'idée générale est de dénier toute communauté avec l'Autre proche. Ainsi, les plus vieux documents écrits slaves orientaux ne peuvent plus être considérés comme communs aux Russes, Biélorusses et Ukrainiens si l'on affirme que le célèbre Dit de la campagne d'Igor ou la Grammaire de Meletij Smotrickij (Vilna, 1619) sont écrits en vieil-ukrainien ${ }^{(8)}$.

(7) A partir d'ici les choses se compliquent singulièrement, car nos auteurs instrumentalisent le modèle polygéniste pour affirmer la pureté de l'ukrainien, tout en appliquant au russe et au biélorusse la théorie de la corruption, ou dégradation à partir d'un rameau initial qu'ils semblent distinguer du rameau originel ukrainien, sans que cela soit vraiment clair.

(8) La Grammatika de Meletij Smotrickij est appelée «grammaire du vieil-ukrainien » déjà à la période soviétique en Ukraine (Nimčuk, 1985), « grammaire du vieux-biélorusse » en Bié- 


\section{Ukraine}

- Un peuple tel que les Slaves n'a jamais existé ; il n'existe que des peuples slaves concrets. (Gubernačuk, 2002, p. 20)

- Ce qu'on croit être une parenté langagière des peuples nommés Slaves est seulement la conséquence des contacts linguistiques (ibid., p. 23)

- Pendant 200 ans on a présenté les Ukrainiens sous une identité anti-historique, les «Slaves ». Or nous, les Ukrainiens, nous avons toujours été des Ukrainiens, des Rusy, des Ukry, des Antes, des Skolotes (scythes), des Aryens mais jamais des Slaves. (Plačynda S., cité par Gubernačuk, 2000, p. 26)

\section{Slovénie}

- Depuis quand les Slaves sont-ils nos frères? Nous les Slovènes avons des ennemis auxquelles nous devrions dire « frères »! (Tomažič, 2003, p.100)

- Pourquoi la langue slovène n'a-t-elle pas eu d'emprunts serbes jusqu'à l'époque de la Yougoslavie ? - parce que nous n'avons pas de racines communes avec les autres Slaves du Sud (Verbovšek, 2004, p. 94).

- Pourquoi le peuple slovène n'a-t-il jamais su, qu'il a des frères dans les Balkans? Parce que il détestait tous les peuples des Balkans. (ibid.)

Voici des exemples parallèles de négation d'une communauté slave :

- Chaque ethnos européen peut répéter ce que les Basques disent souvent : nous venons nous-mêmes de l'âge de pierre (Gubernačuk, 2002, p. 17)

- La langue ukrainienne est non seulement plus ancienne que toutes les autres langues slaves y compris le soit-disant vieux slave mais également plus âgée que le sanscrit, le grec, le latin et les autres langues aryennes (Gubernačuk, 2002, p. 197, se référant à Krasus'kyj, 1991.

- Le sanscrit n'est pas la langue-mère de l'ukrainien, comme on dit parfois, ce n'est pas le sanscrit qui a donné naissance à l'ukrainien mais bien le contraire. (Gubernaèuk, 2002, p. 192)
- La langue et la nation slovènes existent depuis le mésolithique; nous avons gardé notre organisation sociale depuis la préhistoire. (Verbovšek, 2004, p. 173).

- Le latin s'est développé à partir de la langue pré-slave (Tomažič, 2003, pp. 10$15)$.

- La langue slovène est la plus archaïque (ibid., p. 6)

- Les Slovènes sont les seuls héritiers de la culture de l'Europe préhistorique (ibid.)

\subsection{Evolution linéaire de la langue}

Comme dans les théories créationnistes en biologie, les langues, dans la linguistique du nouveau paradigme, existent, inchangées, de toute éternité, ou au moins depuis la préhistoire :

On peut représenter de la façon suivante les conflits de modèles tels qu'on les a jusqu'ici rencontrés :

lorussie actuelle (Jaskevič, 2001) et «grammaire vieux-russe » en Russie (Kuz'minova, 2000). En fait, elle décrivait un état de langue livresque du slavon localisé dans le Grand Duché de Pologne-Lithuanie, avec des formes à demi inventées pour se couler dans les cadres des paradigmes morphologiques de la grammaire latine de Varron, $c f$. Sériot, 2007. 


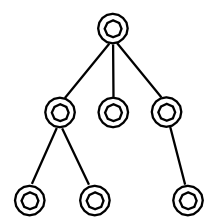

Divergence à partir d'un ancêtre commun (arbre généalogique : ex. : A. Schleicher)

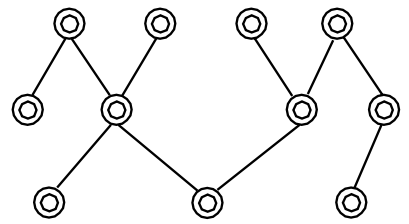

Maillage complexe

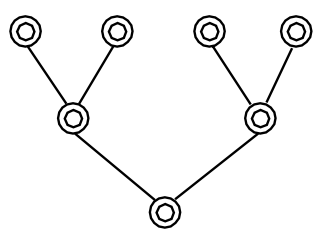

Convergence spatio-temporelle (ex. : R. Jakobson et N. Troubetzkoy)

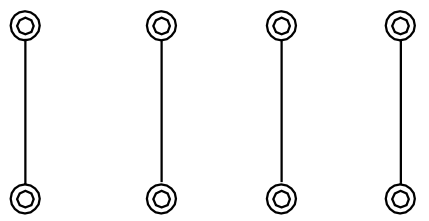

« Nouveau paradigme » (identité spatiale au cours du temps)

\section{La preuve par le nom}

\subsection{Une sémiotique cratylienne}

L'argument de base de la linguistique du nouveau paradigme est que le nom est la preuve de la chose. La méthode n'est guère sophistiquée : on choisit une langue, supposée être très ancienne, qui sert de filtre d'interprétation, ou de métalangue, à des mots actuels, souvent des toponymes (rivières, villes, montagnes), dont la ressemblance " frappante » avec les mots de la langue ancienne (elle-même souvent mal connue) est supposée en donner une clé d'interprétation. Tout est alors censé s'éclairer, devenir transparent, dans un mouvement de révélation, de dévoilement de ce qui était jusque là caché. On peut résumer cette approche à partir de trois principes :

1) l'obscur en dit plus que le clair

2) le vrai est caché sous l'apparence

$3)$ toute ressemblance, même approximative, est signifiante : elle est la preuve d'une identité de sens.

La crypto-sémiotique à la base de ces textes mérite d'être mise en évidence : toute forme a un sens parce que toute forme est nécessairement un signe. Ce type particulier de cratylisme ne s'appuie pas sur un lien naturel entre les mots et les choses, puisqu'il repose sur un relativisme total. En revanche il partage avec les grandes utopies cratyliennes le refus de l'arbitraire du signe, il pratique une remotivation incessante, retrouvant l'inlassable nostalgie du lien direct entre les mots et les choses, remplacé ici par le lien entre les faux mots (opaques) et les vrais mots (transparents). Il s'agit du refus classique d'admettre la perte du lien primordial entre forme et sens.

C'est dans le domaine de la toponymie et de l'étymologie que les promoteurs du nouveau paradigme trouvent leurs principaux appuis.

\section{Slovénie}

Identifiant les Slovènes aux Vénètes, les textes slovènes du nouveau paradigme cherchent à identifier non pas des racines, mais des morphèmes slovènes dans les noms des fleuves, des montagnes et des villes un peu partout en Europe actuelle ou antique. Le slovène devient ainsi la métalangue d'interprétation générale des noms 
de lieux européens, preuve d'un substrat général slovène, grâce à des reconnaissances morphématiques très approximatives.

- Ravenna $=$ Ravna $[$ «égale »];

- Wien = en latin Vindobona, à décomposer en Vinden et bona, nom d'une ancienne colonie, à comparer avec la ville de Koštabona en Istrie ;

- Bodensee (Lac de Constance) : boda = voda [" eau» en slov.]. En pays alémaniques, voda s'est altéré en boden ou baden, d'où de nombreux lacs avec ce nom en Suisse et en Autriche. Il en va de même pour la ville de Budapest: buda $=$ voda

- Le mot log est d'origine slovène ["pré »], et on le trouve partout en Europe. Ce sont des villes et des villages qui datent de la période glaciaire, parce qu'il s'agit d'une signification « originaire »: Logo, Logiano, Loch, Lochen, Lug, Lueg, Lugano, Lugendorf;

- tema [《obscurité »] est un mot slovène à la base de nombreux toponymes d'Europe : en Slovénie : Timava, Temenica, en Allemagne : Temmen, Temning, Demnitz, Timmenrode. Le latin l'a emprunté en Timaus;

- straža [«garde»]. En Slovénie : Straža, Stražišče, ailleurs : Strasbourg ;

- le nom originel de Trieste est Trije ste [«vous êtes trois»]. Les Italiens disent Trieste, sans savoir pourquoi ${ }^{(9)}$.

Tous ces textes professent un mépris total de toute filiation génétique. On pourrait y voir une sorte de post-modernisme s'il ne s'agissait que de dérision par la méthode des calembours. Or il n'est pas question d'y voir une quelconque malice : les décompositions morphématiques sont pour eux une preuve sérieuse. Dans son livre de 1998, T. Grohar propose des étymologies dans un style proche de celui de N. Marr, pour prouver que la langue slovène est la plus ancienne et à l'origine de toutes les langues.

- Ainsi, les noms pour le nombre 1 en serbe JEDAN et en anglais ONE ont pour origine ONJE DAN [ «il est donné»]. Les Anglais ont jeté le mot DAN et ont gardé ON JE, qui est ensuite devenu ONE, qui en slovène se lit VAN, ce qui est familier pour les Slovènes, parce qu'ils écrivent ON et lisent VON ou UN. L'auteur ajoute qu'en français et en italien les mots UN ert UNO sont d'origine slovène.

- HEIMAT = JE MAT [《c'est la mère »]; HERTZ = JE SRC [《c'est le cœur »], GESTERN = JE STAR EN [ « c'est un vieux »], BRUDER = VER U DAR / VERUJ V BOŽJU DAR [《crois dans le don de Dieu », allusion à Abel et Caïn].

- FRAU vient du slovène V RAU ou plus exactement V RAJU [《 en paradis »]. Les Anglais ont dit aux nourrices V U MEN, déformation de V USTA MEN [" dans ma bouche »]. Aujourd'hui ils disent VUMEN et écrivent WOMAN. Quant au pluriel, prononcé WIMEN, il s'agit simplement du nom slovène pour le « pis » (VIMEN)...

- Si l'on compare le mot allemand SEE avec le slovème JEZERO [« lac »], on voit que See et ze sont communs, mais que See est une abréviation de jezero. Chez les Alemands, il manque JE et RO.

- Le mot slovène KOLO [« roue »] est le même qu'en proto-sino-tibétain, qui a la racine $*$ kolo, qu'on ne trouve dans aucune autre langue indo-européenne ${ }^{(10)}$.

- En slovène «bateau » se dit ČOLN, en chinois ČOU.

- En slovène « mouton» se dit JAGNJE, en chinois JANG.

(9) Ces citations sont tirées de Tomažič, 2003, Verbovšek, 2004 et Grohar, 1998. Curieusement, les auteurs omettent d'insister sur le fait que Trieste se dit en slovène Trst, ce qui infirme la décomposition en trije ste.

(10) Il est à noter que *kolo est une racine commune à toutes les langues slaves, P.S. 


\section{Ukraine}

Les auteurs ukrainiens du nouveau paradigme utilisent la toponymie et l'étymologie pour prouver qu'à partir du IV $\mathrm{IV}^{\mathrm{e}}$ siècle avant notre ère un grand mouvement de migrations à partir de la rive droite du Dniepr a développé de très grandes cultures dans les pays d'arrivée. Ainsi, pour Gubernačuk, en sanskrit, langue littéraire des Indo-Aryens, il y a des centaines de mots dont la forme peut être expliquée par une analyse à partir de mots ukrainiens, apportés en Inde par des migrants venus de la région du Dniepr.

- le Nil, fleuve d'Egypte, du sanscrit nila [«bleu foncé »], = Nyl, Nila (prénoms ukrainiens).

- Boguslav, Perejaslav, Ukroslav (villes ukrainiennes) correspondent à Bagušravas, Parjašravas, Ugrašravas (villes des Aryens mentionnées dans les Veda, Gubernačuk, 2002, p. 189).

- Jamuna (rivière en Inde) provient du mot ukrainien Jama [« fosse »].

- Ukhraiïa (nom utilisé dans les Veda) signifie Ukraine, les Indo-Aryens parlaient du pays des Ukry, c'est-à-dire des Ukrainiens.

- Le Roussillon, dans le sud de la France, est à rapprocher du mot RUS'.

- Milano,ville d'Italie, vient du mot ukrainien MYLYJ [« chéri, gentil»].

- Lugo, ville d'Espagne, provient du mot LUG [" pré »]. C'est le même exemple employé par les auteurs slovènes (LOG signifie aussi «pré » en slovène). Jamais cette concurrence n'est notée par les protagonistes, puisqu'ils ne s'intéressent qu'à leur propre discours, et ne travaillent jamais en comparaison sauf pour déconsidérer celui de leur Autre (les Russes pour les Ukrainiens, les Serbes et les Autrichiens pour les Slovènes) ${ }^{(11)}$.

Ce que les promoteurs du nouveau paradigme, dans leur rhétorique sensationnaliste, ne disent jamais, c'est que la quasi-totalité de leurs « découvertes » se trouvent déjà depuis fort longtemps dans tous les discours du ressentiment en Europe orientale. Le slavophile russe Aleksej Khomjakov (1804-1860), cherchant à prouver la suprématie des Slaves sur les Germains, dans son Histoire, mélange de philosophie romantique et d'étymologies fantaisistes, cherche les traces de l'ancienneté du peuplement slave en Europe dans les toponymes. Les Slaves sont les Vénètes, dont on trouve le souvenir en Vendée et dans le Roussillon. La ville de Cahors s'explique par décomposition à partir de racines slaves : Kogorie, et le Périgord est Prigorie («Piémont»). Comme pour les auteurs slovènes, les Vénètes ont fondé Venise (Venezia), et comme les Slaves s'appellent également Antes, Antibes ne saurait être qu'une ville d'origine slave. Les Anglais eux-mêmes avaient été autrefois une tribu slave puisque leur nom English est une variante du nom de la tribu des $\operatorname{Ugliči~}^{(12)}$.

On pourrait ne jamais finir de citer ce pittoresque catalogue d'étymologies fantaisistes et d'interprétations délirantes, mais on se contentera d'évoquer un des textes les plus extrémistes du nouveau paradigme en Ukraine, condensé des procédés précédemment cités, où la forme des mots est la preuve ontologique de l'existence des choses.

J. Kanygyn est docteur en économie, donc avec une formation éloignée de l'histoire et de la linguistique. Pourtant ses livres se trouvent dans les librairies en

(11) Ces exemples sont tirés de Gubernačuk, 2002, pp. 177-207, qui cite également Nalyvajko, 2000, pp. 187-191 et Kobyljux, 2001,pp. 191-192.

(12) L'Histoire d'A. Khomjakov a été publiée après sa mort dans le V volume de ses Euvres complètes (Moscou, 1900-1914). Les citations proviennent des pages 92-93. Sur la linguistique fantastique de Khomjakov, $c f$. Riasanovsky, 1965, chap. IV. 
Ukraine au rayon d'histoire ou de culture. Il pense que les Ukrainiens sont un peuple très ancien, direct descendant de Japhet, fils de Noé. Dans son livre Les vestiges de l'histoire ancienne. Russie-Ukraine (2001), il affirme que le centre de la culture slave, voire indo-européenne, est la Galicie ${ }^{(13)}$. Toute sa théorie repose sur la similitude phonique entre Galicie et Galilée. Gallois, Gaulois, Celtes, Galiciens ne sont que différentes formes de la même dénomination, laquelle est la base étymologique de noms tels que Galaţi (ville de Roumanie), Halstadt (Allemagne), Galice (Espagne). Tous ces lieux ont été formés par des migrations à partir de la protoUkraine. Il est parfois difficile de comprendre qui est l'ancêtre et qui est le descendant, mais une chose est claire, c'est que Jésus-Christ était ukrainien ${ }^{(14)}$.

Il est certain que la théorie de Kanygyn est unanimement rejetée par les historiens et linguistes professionnels, mais l'auteur souligne dans sa préface qu'il ne travaille pas dans le cadre de l'histoire mais dans celui de l'historiosophie, qu'il aide le lecteur à se poser des questions sur les faits historiques. Quoi qu'il en soit, le livre est bien connu du public et très bien vendu en librairie. La société ukrainienne en quête d'identité a besoin de se nourrir de mythes.

\subsection{Le mythe basque}

Avec l'étrusque, le basque est un immense réservoir de fantasmes linguistiques pour les auteurs du nouveau paradigme, dans de nombreux pays. Les similitudes entre le slovène et le basque, par exemple, seraient la preuve de leur unité ancienne et de leur existence à une époque ayant précédé l'arrivée des Indo-Européens en Europe.

Voici des exemples de parallèles basco-slovènes :

\begin{tabular}{|l|l|l|l|}
\hline basque & slovène & basque & slovène \\
\hline goritu & goreti (brûler) & metatu & metati (jeter) \\
\hline masitu & mazati (lubrifier) & magitu & $\begin{array}{l}\text { migati } \\
\text { (faire des mouvements) }\end{array}$ \\
\hline hiratu & hirati (dépérir) & garraitu & garati (trimer) \\
\hline erditu & roditi (naître) & oratu & orati (labourer) \\
\hline zoritu & zoreti (mûrir) & aritu & uriti (exercer) \\
\hline
\end{tabular}

Il est temps maintenant de prendre un peu de recul devant cette avalanche de délires. Et de se rendre compte que le discours sur la singularité absolue a ceci de particulier d'être très commun... La question du basque nous met sur la piste de la matrice commune que nous cherchions au départ.

En 1886 l'abbé Henri Boudet, curé de Rennes-les-bains, dans l'Aude, écrivait une œuvre monumentale : La vraie langue celtique et le cromleck de Rennes-lesBains, dans laquelle il tentait de déchiffrer la totalité des mots basques et occitans grâce au «celte » (en fait, la langue anglaise). 310 pages d'étymologies délirantes, de calembours imparfaits ou géniaux pour montrer que toute langue peut devenir la métalangue explicative de toute autre. En voici quelques savoureux exemples.

Les Occitans étaient autrefois des chasseurs de marsouins : hog-sea (hogsi), marsouins, - to hit, frapper, hand, la main $\rightarrow$ hogsihithand : occitan (p. 143).

(13) La Galicie est une ancienne province de l'Autriche-Hongrie, regroupant la région de Cracovie (en Pologne actuelle) et de Lviv (en Ukraine actuelle).

(14) Notons qu'une des thèses du Mythe du vingtième siècle (1930) d'Alfred Rosenberg, théoricien nazi, était que Jésus était «Aryen », et non pas juif. 
Les noms de mois en basque sont remotivés à partir de l'anglais :

- «Urtharrilla [“janvier”] : le mauvais temps du mois de janvier arrête les travaux de ceux qui voudraient passer la herse dans leurs champs : - to hurt, "nuire", — to harrow, "herser", — to will "vouloir" " (p. 120).

Il en va de même de toute expression idiomatique ou de toute unité lexicale :

- «Le lever du soleil : iruzki sartzea, présente le sens suivant : celui qui est fatigué déteste d'entendre bourdonner dans l'air — to hear (hir), "entendre", - to huzz (heuzz), "bourdonner", — sky (skaï) air, — to hatter, "harasser", to hate, "détester" (p. 122).

- « Ichkilin [“épingle”] : L'extrême propreté était bien loin de briller dans les hôtelleries où s'arrêtaient d'infortunés voyageurs consciencieusement armés d'une épingle : on comprend aisément de quels insectes dégoûtants et agaçants il est ici question, - to itch, "démanger", to kill, "tuer", to inn, "loger dans une auberge"» (p. 124).

La méthode est la même, puisque là aussi l'opaque devient transparent par « analyse » translinguistique. Mais une lecture attentive du texte de l'Abbé Boudet par son préfacier Gérard de Sède permet d'en mettre en avant l'aspect profondément parodique. La méthode de décomposition de l'Abbé Boudet le range parmi les grands logophiles au même titre que Raymond Roussel et Jean-Pierre Brisset ${ }^{(15)}$.

\subsection{De la prétendue originalité du discours sur les origines}

Ce qui se passe actuellement en Europe centrale et orientale n'est pas exceptionnel. On peut faire de nombreux parallèles avec d'autres pays à d'autres époques. Le Japon en fournit un bon exemple extra-européen, avec une même recherche des origines, une même affirmation de singularité absolue, au début du $\mathrm{XX}^{\mathrm{e}}$ siècle. Le but était de tenter à tout prix d'abaisser la culture chinoise et d'en effacer les traces dans l'histoire du Japon. Un historien renommé, Sokitchi Suda, dans son livre La pensée chinoise et le Japon (1933-1936), prétend que la pensée chinoise, acquise au Japon de façon uniquement livresque, et donc artificielle, n'a pas eu d'influence sur la pensée japonaise. Le but est bien de dénigrer l'Autre dont on veut se démarquer et pour cela sont convoqués des jugements sur la langue : pour Suda, la langue chinoise, qui ne fait aucune distinction entre le nom, le verbe et l'adjectif, n'autorise pas une réflexion profonde (Yuki, 1984, p. 156). Un autre historien japonais, Ukitchi Tagutchi, s'appuie sur l'équation classique langue = peuple pour attribuer aux Japonais une origine aryenne. Dans ses Etudes sur l'Antiquité, parues en 1902, il compare la syntaxe de différentes langues et conclut que le sanskrit, le latin et le grec ressemblent plutôt au japonais et au turc qu'aux langues indo-européennes modernes. Par conséquent, ce sont les Japonais qui sont plus directement les descendants des Aryens. Il découle de ce raisonnement que les Européens, en se réclamant d'une origine aryenne, arrachent aux Japonais leurs ancêtres véritables (Yuki, ibid., p. 158). On reconnaît la thématique du nom volé chez les auteurs ukrainiens actuels : les « Moscovites » (nom donné aux Russes par les nationalistes ukrainiens) auraient volé aux Ukrainiens leur véritable nom de Russes, ne leur laissant d'autre solution que de se trouver un autre nom, faute de mieux (Ukraine signifie « bord », « extrémité », c'est-à-dire « marche »).

(15) Sur les logophiles, cf. Pierssens, 1976. 


\section{Conclusion}

On peut maintenant faire le bilan de la lecture, à la fois ébouriffante et épuisante, de ces textes fantastiques.

Les mythes nationalistes identitaires, à certaines époques de constitution politique de collectivités humaines, sont acceptés par le commun des mortels comme par les savants. Phénomène remarquable : les scientifiques ethnicistes font preuve d'une loyauté absolue envers leur communauté ethnique d'appartenance. Même le grand linguiste serbe Pavle Ivić a apporté sa pierre à la construction du mythe linguistique national serbe (Ivić, 1971). Il ne semble venir à l'idée de personne qu'une démarche qui se veut scientifique pourrait commencer par confronter des arguments, encore moins s'appuyer sur une méthode hypothético-déductive. Mais il n'y a pas de risque de faire une découverte : la réponse est donnée d'avance, avant même que toute question soit posée. La linguistique du Nouveau paradigme ne fait pas d'hypothèse, elle ne pose que des affirmations.

L'objet qu'il reste à construire est la crypto-linguistique, sur le modèle de la crypto-histoire. Il est très vraisemblable qu'on en trouvera les origines dans les affirmations identitaires en Europe occidentale dès la Renaissance puis au XVIII ${ }^{\mathrm{e}}$ siècle. Autochtonisme et protochronisme s'y mêlent à une attitude de refus de la science traditionnelle, à une revendication de nouveauté totale, qui ignore ou fait semblant d'ignorer l'histoire de son propre discours chez les proches voisins dont on veut précisément se démarquer. Ces procédés de légitimation reposent sur un imaginaire collectif et une obsession identitaire dont l'étude pourrait être un élément de réflexion dans la construction européenne.

\section{Bibliographie}

Angenot, M. (1996) : Les idéologies du ressentiment, Montréal : XYZ.

AurouX, S. et al. (1983) : La linguistique fantastique, Paris : Denoël-Clims.

BogdANOv, A. (1907) : Krasnaja zvezda : roman-utopija; trad. fr. : L'étoile rouge; L'ingénieur Menni : romans utopiques, Lausanne : L'Age d'Homme, 1985.

Boudet, H. (1866) : La vraie langue celtique et le cromleck de Rennes-les-bains, Carcassonne : Pomiès ; rééd. : l'Eil du sphinx, Paris, 2006.

FILIN, F. (1972) : Proisxoždenie russkogo, ukrainskogo i belorusskogo jazykov, Leningrad : Nauka. [L'origine des langues russe, ukrainienne et biélorusse.]

GROHAR, T. (1998) : Zgodovina v luči jezikovnih sledi, Radovljica. [L'histoire à la lumière des traces linguistiques.]

GUBERNAČUK, S. (2002) : Jak gul stolit', jak šum vikiv-ridna mova, Kyïv : Blic inform. [Comme le bruit des siècles : la langue maternelle.]

IVIĆ, P. (1971) : Srpski narod i njegov jezik, Beograd : Srpska književna zadruga. [Le peuple serbe et sa langue.]

KANYGYN, J. (2001) : Vihy svjaščennö̈ istorï̈. Rus'-Ukraïna, Kyïv : Ukraïna. [Les vestiges de l'histoire ancienne. Russie-Ukraine.]

JASKEVIČ, A. (2001) : Starabelaruskija gramatyki, Minsk : Belaruskaja navuka. [Les grammaires vieux-biélorusses.] 
Khomjakov, A.(1900-1914) : Polnoe sobranie sočinenij, Moscou. [Euvres complètes.]

KoBYLJUX, V. (2001) : «Bez sanskrytu nemaje spravn'joï istoriï Ukraïny », Osvita, 11. [Sans le sanskrit, il n'y a pas d'histoire véritable de l'Ukraine.]

KRASUS'KYJ M. (1991) : Drevnost'malorossiiskogo jazyka, Kyïv : Indojevropa. [L'ancienneté de la langue petite-russienne.]

KuZ'Minova, E. (2000) : Grammatiki Lavrentija Zizanija i Meletija Smotrickogo, Moskva : Izdatel'stvo moskovskogo universiteta. [Les grammaires de L. Zizanij et de M. Smotrickij.]

LÖFGREN, O. (1989) : «The Nationalization of Culture », in National Culture as Process, rééd. de Ethnologica Europea, XIX, 1, pp. 5-25.

MAVRODIn, V. (1946) : Drevnjaja Rus' (proisxoždenie russkogo naroda i obrazovanie kievskogo gosudarstva), Leningrad : Ogiz. [La Russie ancienne (origine du peuple russe et formation de l'Etat de Kiev).]

MicheA, J.-C. (1979) : « Kial venkis esperanto ? », Critique, n³87-388, p. 661674. [Pourquoi l'espéranto a-t-il vaincu?]

NALYVAJKO, S. (2000) : Tajemnyci rozkryvaje sanskryt, Kyïv. [Les mystères sont éclaircis par le sanskrit.]

NIMČUK, V.V. : Movoznavstvo na Ukraïni v XIV-XVII st., Kyïv : Naukova dumka. [La linguistique en Ukraine aux XIV-XVII ${ }^{\mathrm{e}}$ siècle.]

Pierssens, M. (1976) : La tour de Babil, Paris : Minuit.

RIASANOVSKY, N. (1965): Russian and the West in the Teaching of the Slavophiles. A Study of Romantic Ideology, Gloucester. Mass. : Peter Smith.

RIZNYČENKO, O. (2001) : Spadščina tysjačolit', Odessa. [L'héritage des millénaires.]

SERIOT, Patrick (2006) : «Droit au nom et droit du nom : les Ruthènes sont-ils une minorité ? ", Slavica Occitania, Hommages à Roger Comtet, $\mathrm{n}^{\circ} 22,2006$, pp. 207-233.

- (2007) : « A quelle tradition appartient la tradition grammaticale russe? », Langages, $\mathrm{n}^{\circ} 167,2007$, pp. 53-69.

SMIT, M. de : «Uralists Against History », April 9, 2001 http ://www.geocities.com /isolintu/Uralists_Against_History.htm

THIESSE, A.-M. (2001) : La création des identités nationales. Europe 18e-20e s., Paris : Seuil.

TOMAŽIČ, I. (2003) : Slovenske korenine, Ljubljana: Samozaložba. [Les racines slovènes.]

TroubetZKoy, N. (1929) : «Gedanken über das Indogermanenproblem », Acta linguistica, I, fasc. 2, pp. 81-89. [trad. fr. : «Pensées sur le problème indo-européen », dans P. Sériot (éd.) : N.S. Troubetzkoy. L'Europe et l'humanité. Ecrits linguistiques et paralinguistiques, Liège : Mardaga, 1996, pp. 211230.

VERBOVŠEK, L. (2004) : Danes, iz včeraj za jutri, Ljubljana : Jutro. [Aujourd'hui, d'hier à demain.]

Y UKI, A. (1984) : « Mémoire sociale et nationalisme au Japon », Le Genre humain, $\mathrm{n}^{\circ} 11$ : La société face au racisme, pp. 153-178. 